\title{
Impact of Face Partitioning On Face Recognition Performance
}

\author{
Harihara Santosh Dadi, Krishna Mohan P.G.
}

\begin{abstract}
Face partitioning algorithm is presented in this paper. Face is first divided into face parts namely head, eye pair, left eye, right eye, mouth and nose. Instead of giving the entire face as input in testing and training phases of face recognition algorithm, the face parts are given individually. Eigen features of all the face parts are extracted separately and given to the individual classifiers. Finally the classifier outputs are given to the decision making algorithm. This accepts all the face parts and generates a face based on the algorithm. ORL data base is used for evaluating the performance of this new technique. Results are separately calculated with and without face partitioning technique. Results show that face recognition rate is increased by using the combination of face partitioning technique and PCA. The new algorithm is also verified on 8 different data sets. There is an improvement of $15 \%$ face recognition rate using the new algorithm on ORL database.
\end{abstract}

Index Terms: Face Partitioning, Facial features, Recognition engine, Support Vector Machine, Decision making algorithm.

\section{Introduction}

Face recognition targets at identifying the human being uniqueness by evaluating the facial features with the available face data set features. The face data set, with known distinctiveness, is referred as the face gallery and the input face requiring determining the identity is the probe.

Face recognition has been an important topic of research originated way back in the year 1961. Numerous algorithms are developed on face recognition particularly in the last two to three decades. Improving the Face recognition rate is always the challenge ever since the first algorithm was developed. In 1991, Alex Pentland and Matthew Turk [1] - [4] applied Principal Component Analysis (PCA) which was invented in 1901 to face classification. This has become the standard known as the eigenface method and is today an inspiration for all face recognition algorithms evolved. Sebastain Mike et. al. [5] competing with PCA which is an orthogonal linear transformation proposed Fisher Discriminant Analysis with Kernels (KFD). Being a non linear classification model, KFD has better performance over PCA. The problem with KFD is that it uses all the training samples in the solution not only the difficult ones which makes the algorithm slower and also complex. Hailing Zhou et. al. [6] says that the performance of face recognition systems degrades with the variants like illumination, pose, and facial expressions. In their paper, they proposed 3D face recognition systems which contains 3D facial geometry information. For more 3D facial models refer. It achieves greater recognition accuracy than visual face recognition systems. Though it is insensitive to illumination variations, still it is difficult to apply in the absence of visible light. All these single modal and multi modal face recognition algorithms are having the common problem of poor way of igniting the recognition engine. Gheorghita Ghinea et. al. [7] first made an attempt in integrating the Hausdorff Distance (HD) and Schur decomposition for dimensionality reduction based face recognition. The Schur faces have the high discriminative power and performed well over the standard face recognition methods. Still it is in need of some kind of preprocessing step and an enhanced recognition engine for better face recognition performance. Navneet Dalal et. al. [8] made a paradigm shift by introducing Histogram of Oriented Gradient (HOG) features instead of Eigen faces which are in the standard PCA algorithms. HOG features being dense overlapping grid gives very good results for person detection. HOG features have the advantage of fine orientation binning, fine scale gradient, relatively course spatial binning and high quality local contrast normalization which are important for good performance. The author says that there is still room for optimization. Le An et. al. [9] introduced face recognition in multi camera surveillance videos. They developed unified face image (UFI) by fusing face image from different cameras. This is more effective as it uses multi cameras for face feature extraction from different orientations. This algorithm needs a high experimental setup. Paola Campadelli et. al. [10] developed feature base face recognition. This is an automatic face recognition which localizes the facial features. The author considered 16 fuducial points. This can be more effective if the geometry and position of the intermediate points are also considered. Sasi Kumar Gurumurthy et. al. [11] designed a face recognition system based on lips. By localizing the facial features, the face recognition rate is improved. Ognjen Arandjelovic et. al. [12] achieved robust video face recognition by combining a learnt generic face invariant and weak photometric model. 
In this paper we propose a novel approach for developing face recognition algorithm. Here, we divided the face in to face parts like head, nose, right eye, mouth, left eye, and eye pair. Paul Viola et. al. [13] developed face parts detection algorithms. The features like Eigen are extracted for these parts and given to the classifiers. The classifier compares the features of the probe and the features of the gallery in the database. Each classifier outputs the face part. All these face parts are again given to the decision making algorithm which finally generates the matched face. We compare our algorithm's results with Eigen face feature algorithm. Finally we compare our algorithm with the standard face recognition algorithm, PCA.

While numerous face recognition algorithms are being developed, the authors are comparing them with the existing ones very superficially and few simple comparisons are presented. Given that large set of techniques and the theories that are applicable for face recognition, it is evident that the detailed analysis and bench marking these algorithms is very crucial. Effort done by Universities and research laboratories in developing the data sets pushed the comparisons of face recognition algorithms to the higher level. CMC and ROC curves [14] - [16] were introduced for comparisons. Apart from finding the recognition rate, these curves become the basis for showing the superiority of the author's developed algorithms.

The contributions of this paper are as follows:

- We develop a novel face partitioning algorithm based on localizing the facial features. This works well for finding out the face parts and is more insensitive to the illumination, pose and facial expression variations. As the features are more localized, the variations become substantially reduced when we see for individual face parts.

- We presented a decision making algorithm which accepts different face part outputs from different classifiers and generates a face output.

- Extensive comparisons are made by taking the performance metrics curves namely CMC and ROC and showed that the curves are effective for proposed algorithm.

$\bullet$

\section{Related Work}

Face recognition methods mainly deal with images which are of large dimensions. This makes the task of recognition very difficult. Dimensionality reduction is a concept which is introduced for the purpose of reducing the image dimensions. PCA is the most widely used dimensionality reduction and also for subspace projection. PCA can supply the client with a lower-dimensional picture, a projection of this object when seen from its informative view point. This can be achieved by taking only the starting few principal components in such a way that the dimension of the transformed data is minimized. The linear combination of pixel values here in PCA are called Eigen faces.

Two performance metrics curves are considered. Cumulative Match Score Curves (CMC) is the curve between the rank on the $\mathrm{x}$-axis and face recognition rate on the $\mathrm{y}$-axis. Receiver Operating Characteristics (ROC) is the graph between false acceptance rate and verification rate. ROC curves are more informative

\section{Face Recognition Algorithm}

A typical face recognition algorithm is presented in this section. For any face recognition algorithm, there are two phases. One is training phase and the other is the testing phase. In the training phase, the features of all the faces in the gallery are found and stored in the data base. Eigen features are taken in the sample face recognition algorithm shown below in the figure 1 . In the testing phase, the features of the probe are calculated. These features and the features of the gallery are given to any of the classifier. SVD classifier is taken as example in the figure. The Eigen features of the probe and the Gallery are taken by the SVD. The classifier looks for the closest feature matching face from the gallery with the probe and gives that face as output. Figure 1 shows the sample face recognition algorithm block diagram. 


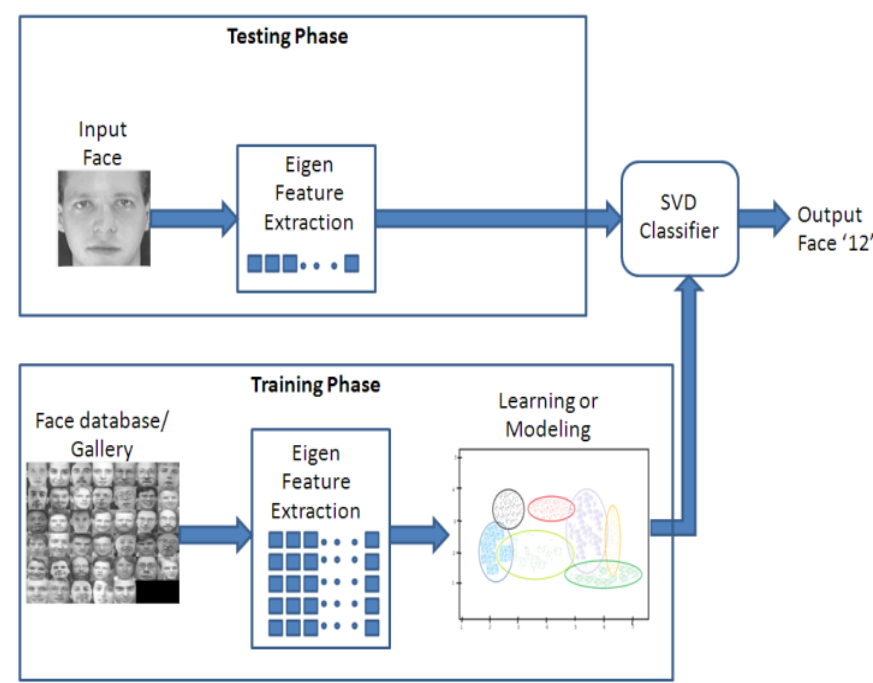

Fig.1 existing face recognition system

\section{A. Face partitioning Algorithm:}

\section{Proposed Method}

The Face image is partitioned in to different parts like Eye pair, mouth, left eye, nose, right eye and head. The face data set is divided in to data sets of these features. Each data set is divided in to testing and training sets. The Eigen features are found for the training data set. These features are given to the SVD classifier. In the testing phase the testing image is also partitioned in to image features mentioned above. Each part of the face is given to the corresponding classifier. The SVD classifier generates the output image for which the Eigen features are closely matched. All the classifiers generate different types of face images as outputs. All these are given as inputs to the decision making algorithm. In this stage, the optimal face is detected and given as output.

\section{1) Partitioning of Faces}

The images in the face data set are divided in to face features. Figure 2 shows the first faces of all the 40 members in the ORL database. Figure 3 shows the block diagram of the face partitioning algorithm used in the training phase. Here, each face image is divided into different parts. Figure 4 shows how the face parts are detected. Head in red, right eye in magenta, nose in blue, left eye in black, mouth in purple and eye pair in green. Figure 5, 6, 7, 8, 9 and 10 are the gallery of heads, mouths, eye pairs, left eyes, noses and right eyes of the first image of all the persons in the ORL database respectively. Figure 11 shows all the 10 face images of the first person in the ORL database. Figure 12 shows how the face parts are detected. Figure 13 shows the block diagram of the face partitioning algorithm used in the testing phase. Figure 14, 15, 16, 17, 18 and 19 are the gallery of heads, mouths, eye pairs, left eyes, noses and right eyes of the all the ten images of the first person in the ORL database respectively. 
2) Gallery images

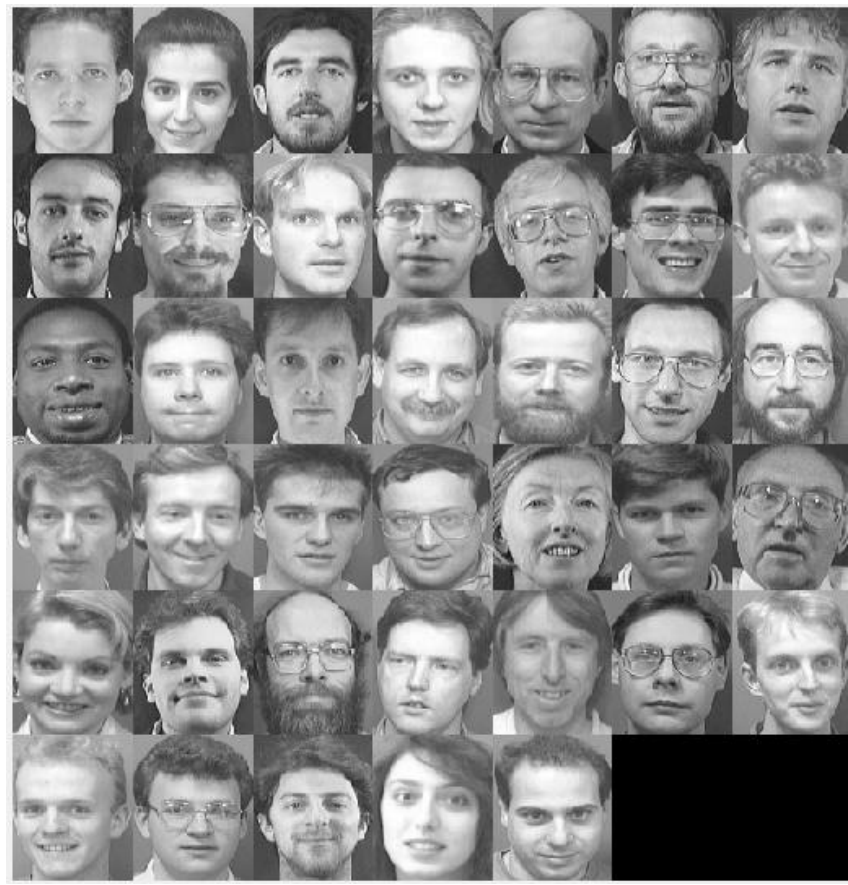

Fig. 2 First face image of all 40 people in the ORL database

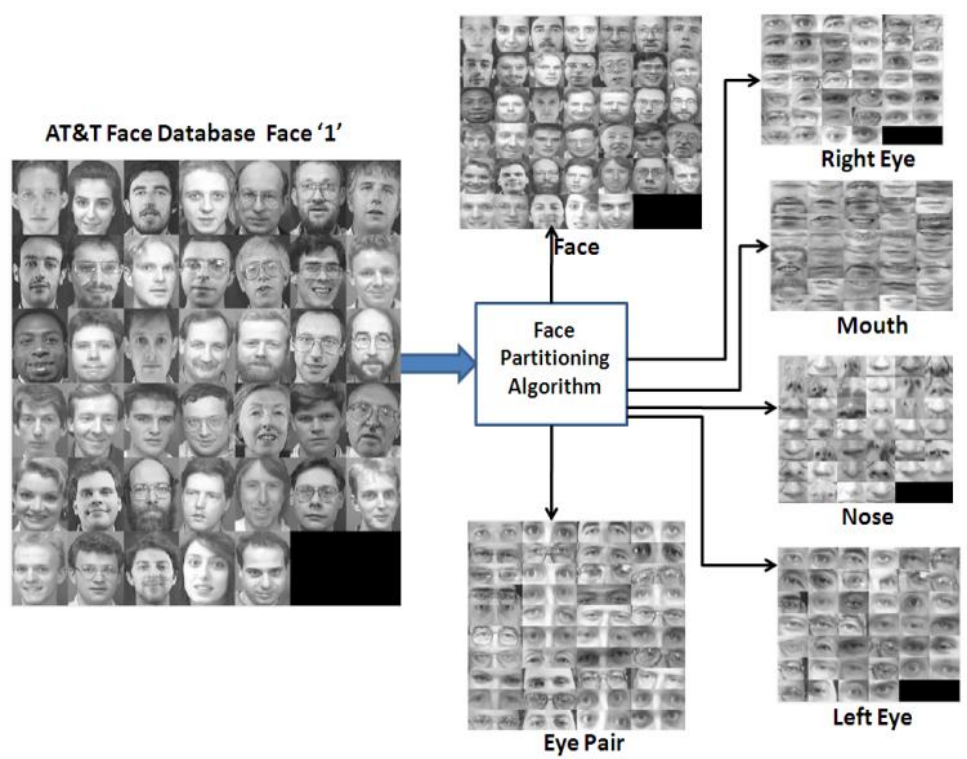

Fig. 3 Face partitioning algorithm in the training phase which partitions the face into head, right eye, mouth, nose left eye and eye pair. 


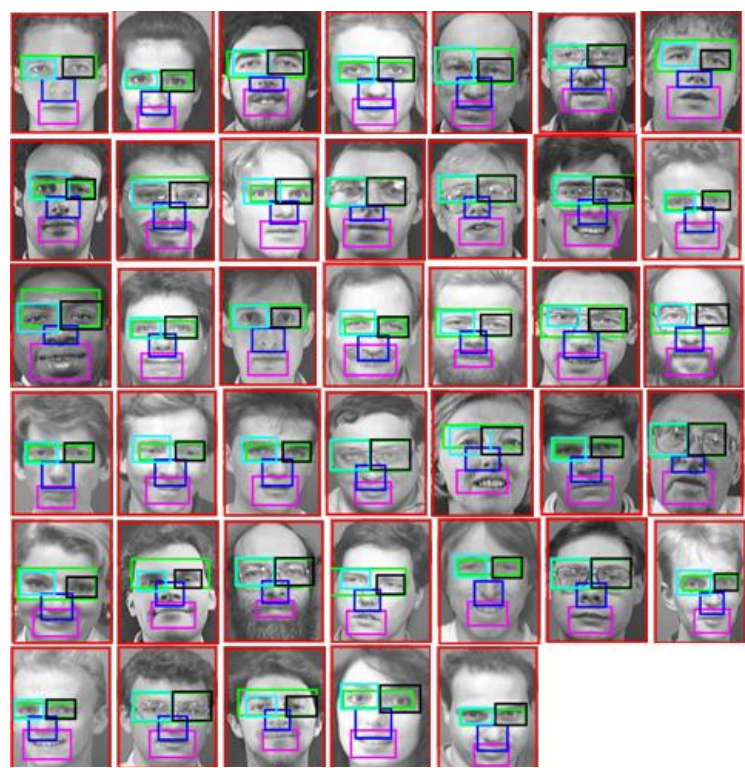

Fig. 4 Face parts shown in different colors. Head in red, right eye in magenta, nose in blue, left eye in black, mouth in purple and eye pair in green.

a. Head Images

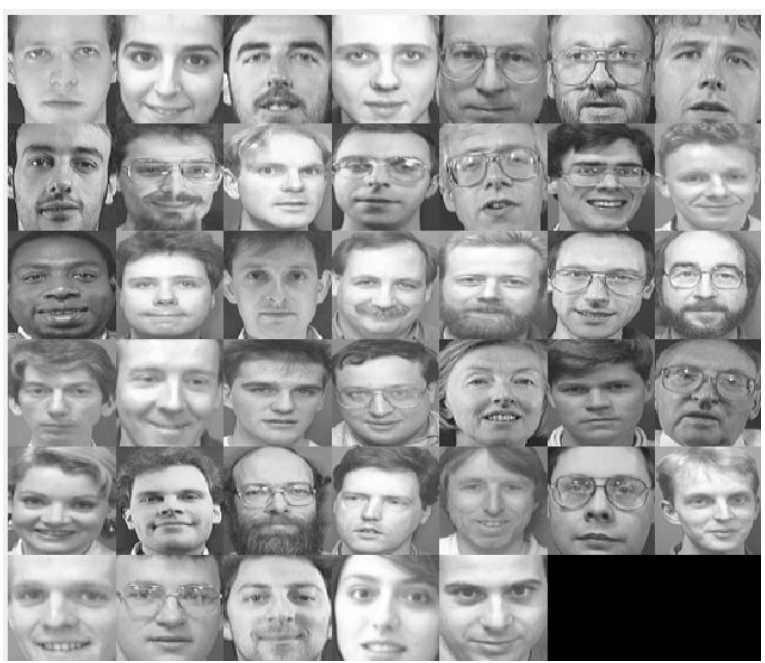

Fig. 5 Head parts of all 40 people from ORL database.

b. Mouth Images

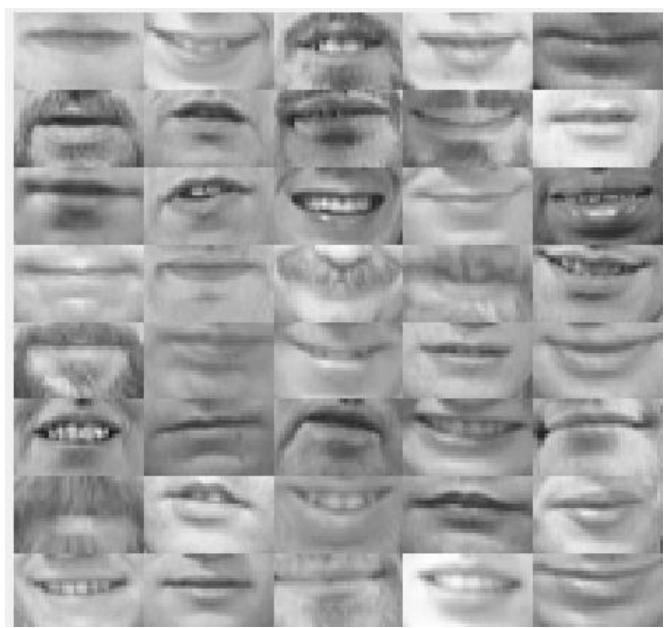

Fig. 6 Mouth parts of all 40 people from ORL database. 
c. Eye Pair Images

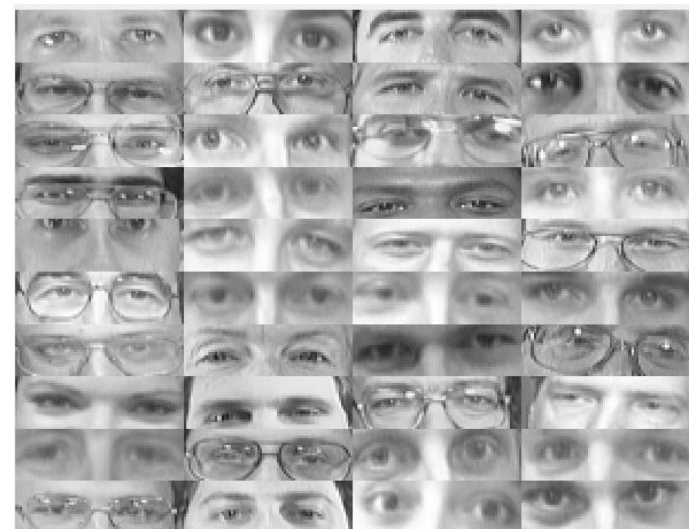

Fig. 7 Eye pair parts of all 40 people from ORL database.

\section{d. Left Eye Images}

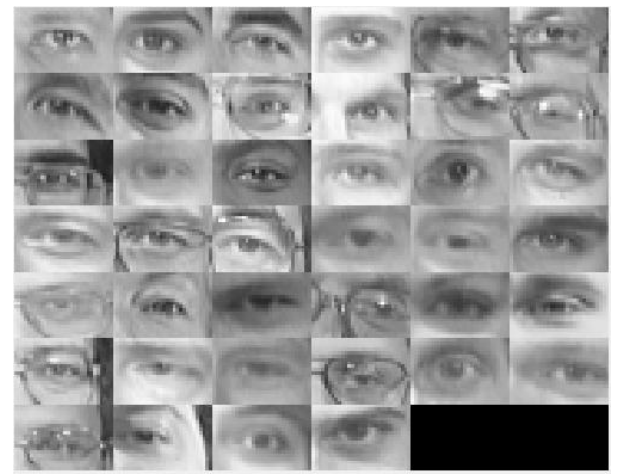

Fig. 8 Left eye parts of all 40 people from ORL database.

\section{e. Nose Images}

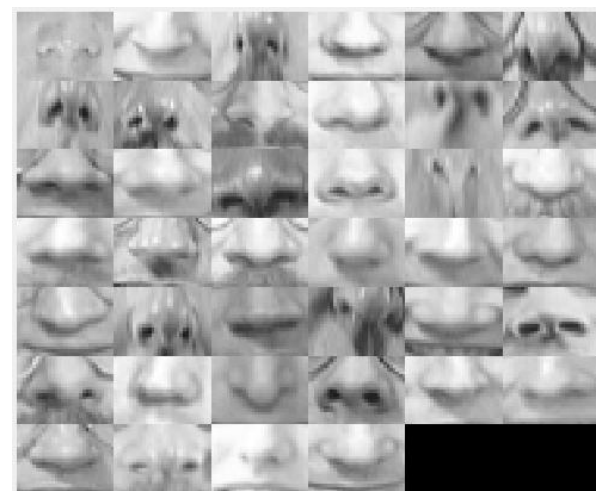

Fig. 9 Nose parts of all 40 people from ORL database.

\section{f. Right Eye Images}

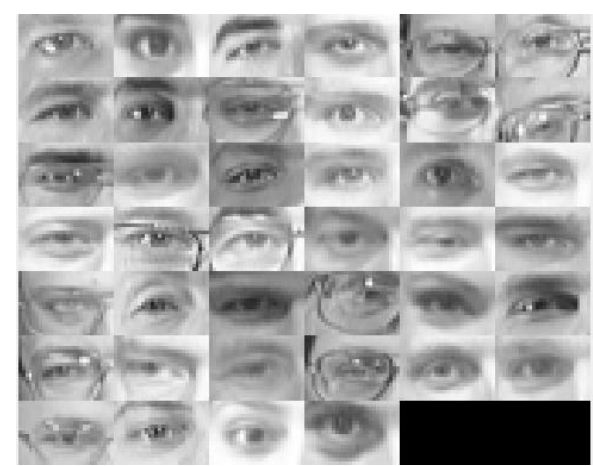

Fig. 10 Right eye parts of all 40 people from ORL database. 
g. Probe Image

The face images of the first person in the AT\&T Database.

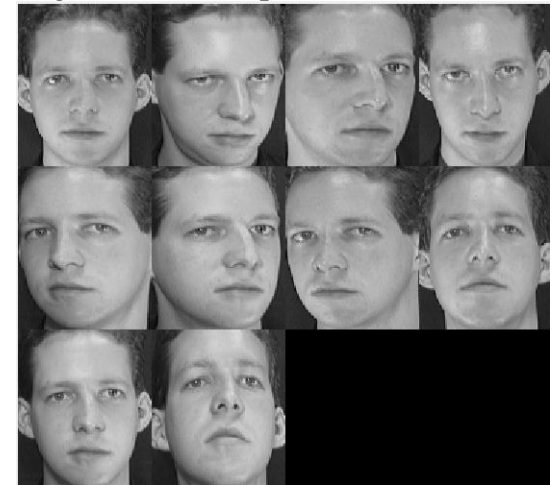

Fig. 11 All 10 images of first person from ORL database.

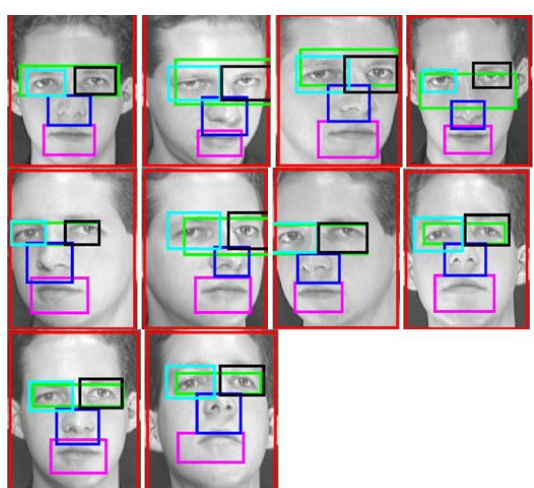

Fig.12 Face parts shown in different colors for the first person from ORL data base.

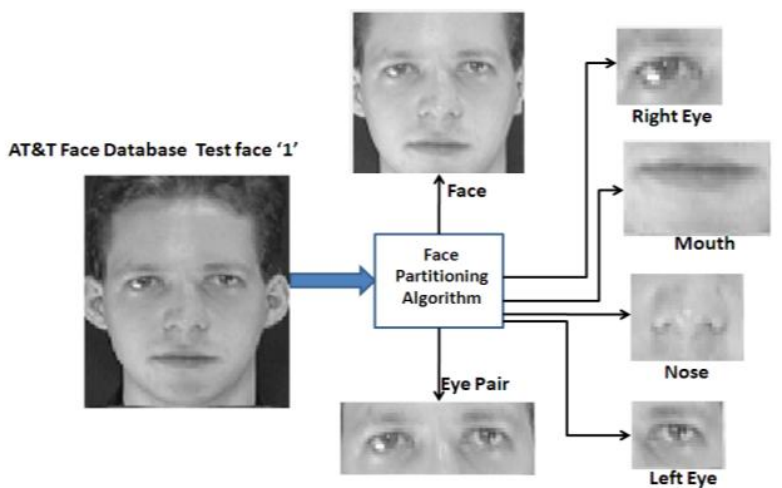

Fig. 13 Face partitioning algorithm which partitions the face image into different parts in the testing phase.

\section{a. Head Image}

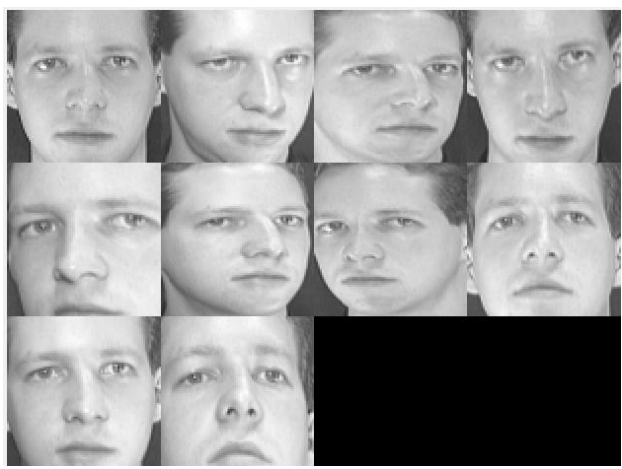

Fig. 14 Head parts of the first person in ORL database 
b. Mouth Image

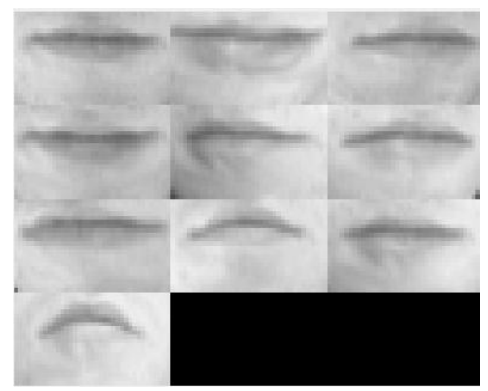

Fig. 15 Mouth parts of the first person in ORL database

c. Eye pair Image

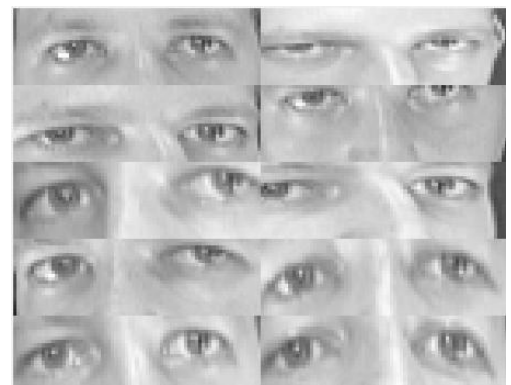

Fig. 16 Eye pair parts of the first person in ORL database

\section{d. Left eye Image}

\section{e. Nose Image}

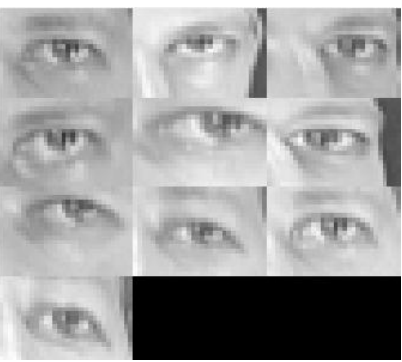

Fig. 17 Left eye parts of the first person in ORL database

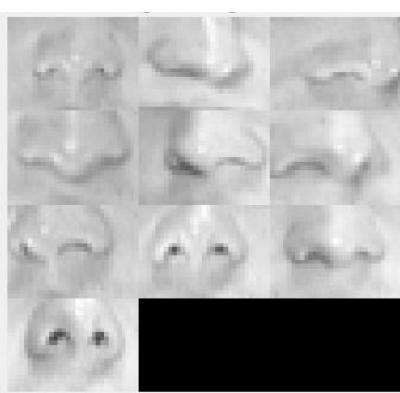

Fig. 18 Nose parts of the first person in ORL database

\section{f. Right eye Image}

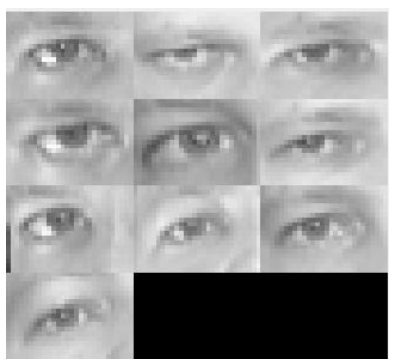

Fig. 19 Right eye parts of the first person in ORL database 


\section{3) Training Phase}

In the training phase, all the face part gallery features are extracted and individually trained by using any classifier. Here we extracted Eigen features and used SVD classifier. Figure 20 shows the training of all the face parts in the training phase. Figure 21 shows the overall training phase of our proposed method. This way of igniting the recognition engine is introduced in this section.
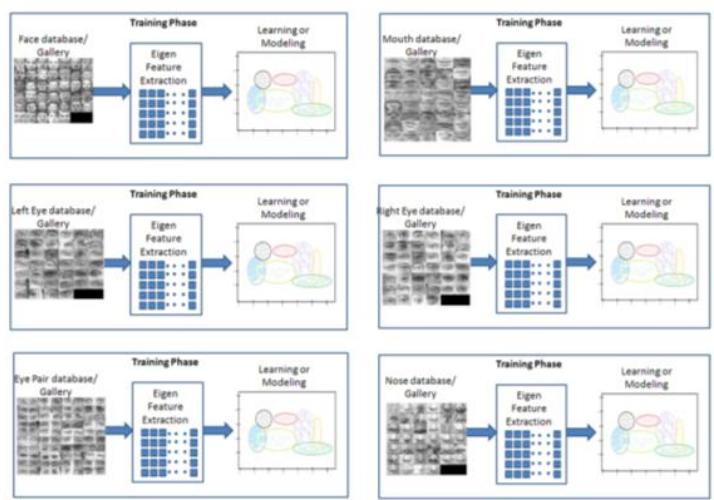

Fig. 20 Training phases of head, right eye, mouth, nose, left eye, and eye pair galleries.

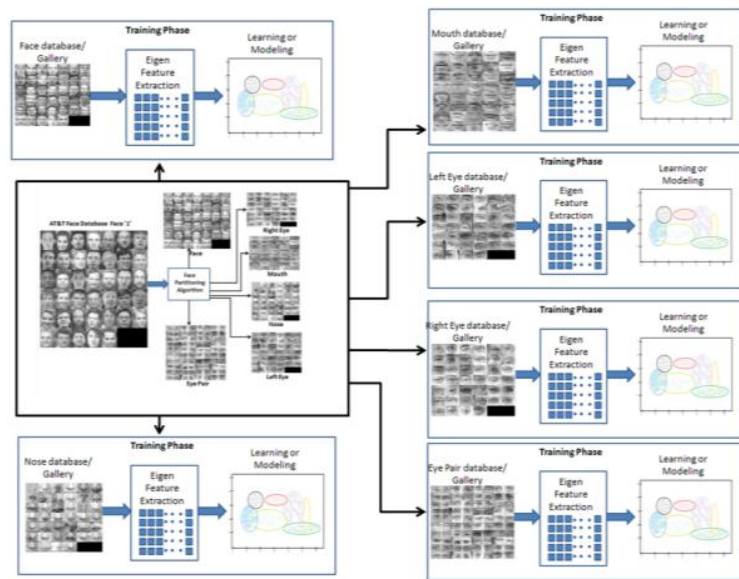

Fig. 21 Overall training phase of the proposed algorithm

\section{4) Testing Phase}

In the testing phase, the probe image is given to the face partition algorithm. The Eigen features of the face parts are extracted individually. Figure 22 shows how the Eigen features of first image of first person in the ORL database are extracted in the testing phase. Figure 23 shows the overall testing phase of the proposed algorithm. Figure 24 shows the face partitioned face recognition algorithm. The training and the testing phases are separately shown.
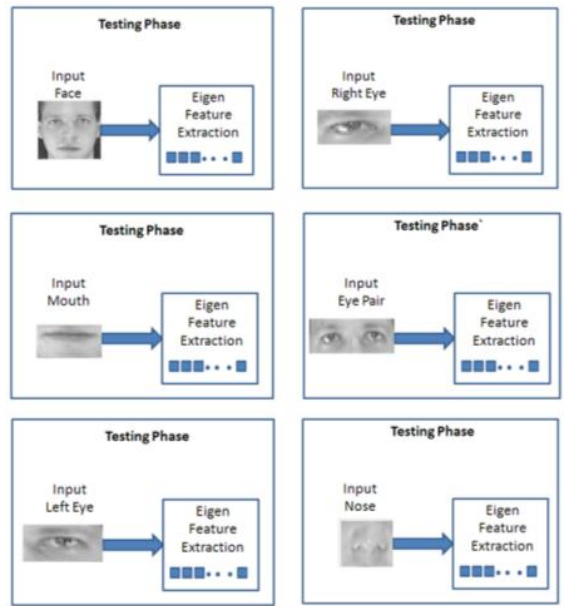

Fig. 22 Feature extraction of face parts of the probe image in the testing phases. 


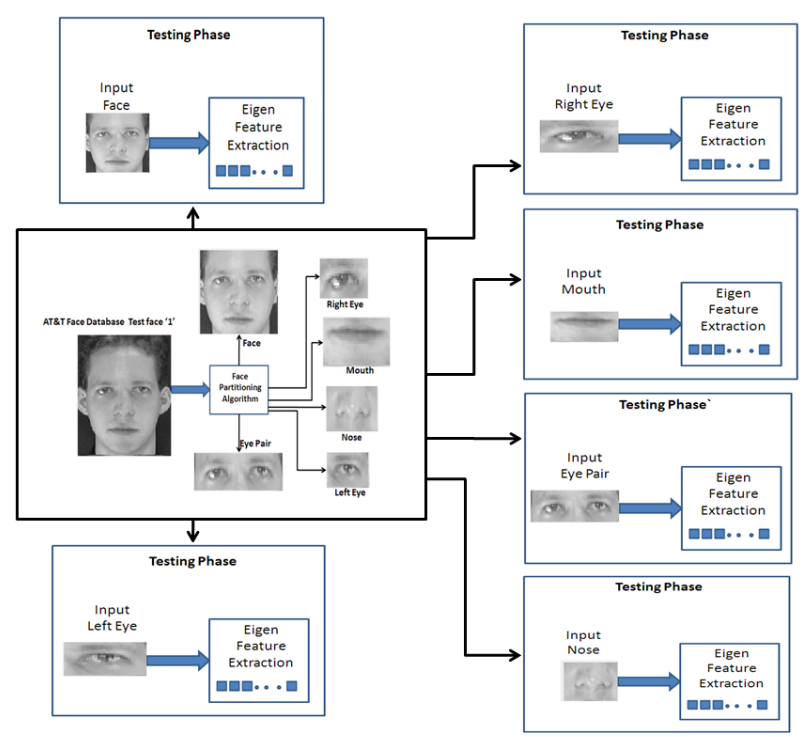

Fig. 23 Overall testing phase of the proposed algorithm.

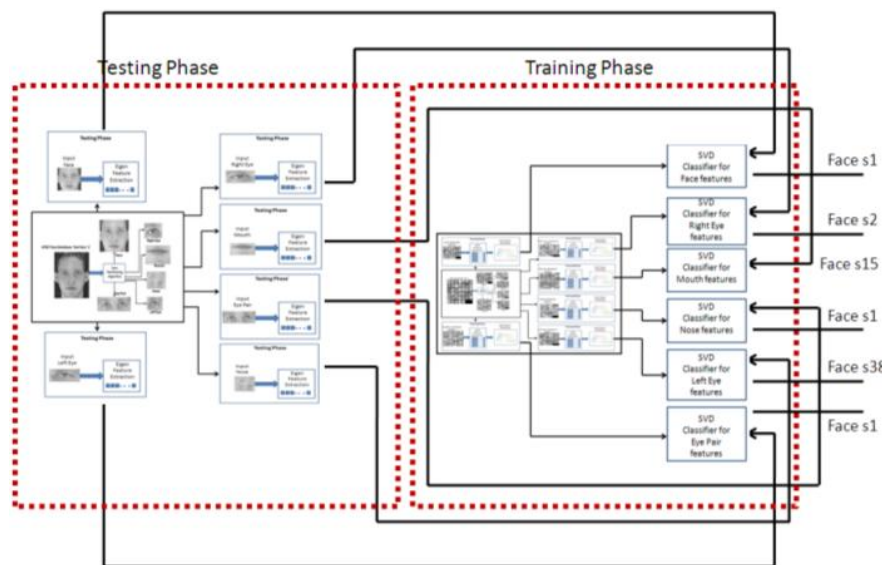

Fig. 24 Face Partitioned Face Recognition System testing and training phases.

\section{B. Decision making algorithm:}

Let there are ' $n$ ' classifiers for different face partitioned datasets. Each classifier compares the features of the gallery and the features of the probe. The classifier outputs the nearest face part from the gallery with the probe. The input to the decision making algorithm are the outputs from the SVM classifiers. Decision making algorithm compares all the face parts. The face with more number of face parts is produced as output. Here in our algorithm the face with more than two face parts is considered as the output of the decision making algorithm. Figure 25 shows the complete face partitioned face recognition system.

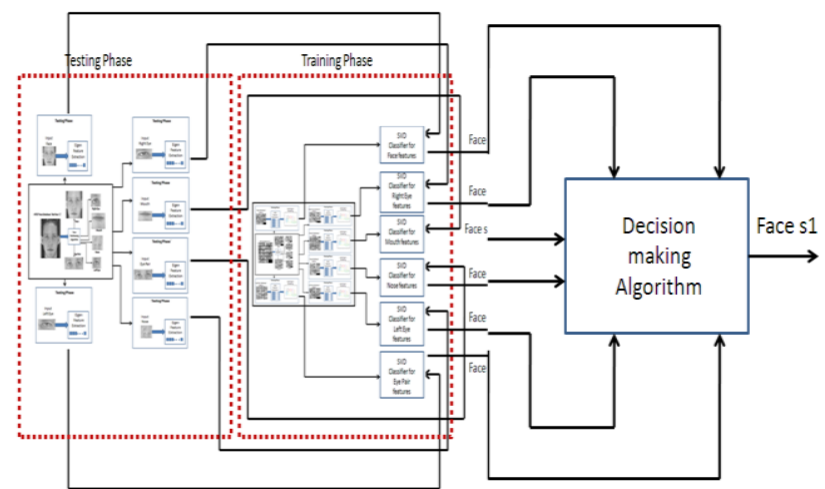

Fig. 25 Face Partitioned Face Recognition System. 


\section{Experimental Results}

Experiments have been conducted on proposed algorithm by taking ORL AT\&T data base [17]. For training phase the first eight face images are taken and for the testing purpose the last two face images are taken. The face database is first divided in to six separate databases namely head, eye pair, left eye, mouth right eye and nose. Eigen features are extracted in the training phase for all the data sets. And in the testing phase, the Eigen features of these parts are taken and given to the SVD classifiers parallel. The outputs of these classifiers are again feed to the decision making algorithm. The output of decision making algorithm is the output of the proposed system.

The individual results are shown for the first test images in table I. The results for different data sets are shown in different columns. The last column is the proposed method. The green color indicates that the proposed algorithm is generating the correct output where as the original PCA algorithm is giving the wrong result. The red color indicates that even by using the proposed algorithm still some of the face images are not showing the correct output. There are 80 test images and 320 train images. Out of 80 test images, 64 images are correctly recognized by the PCA algorithm. Whereas by using the proposed algorithm, 76 test images are correctly recognized. There is an improvement of $15 \%$ in face recognition rate when compared with the PCA algorithm on ORL database.

Figure 26 shows the graph between testing versus training of test face image 1.

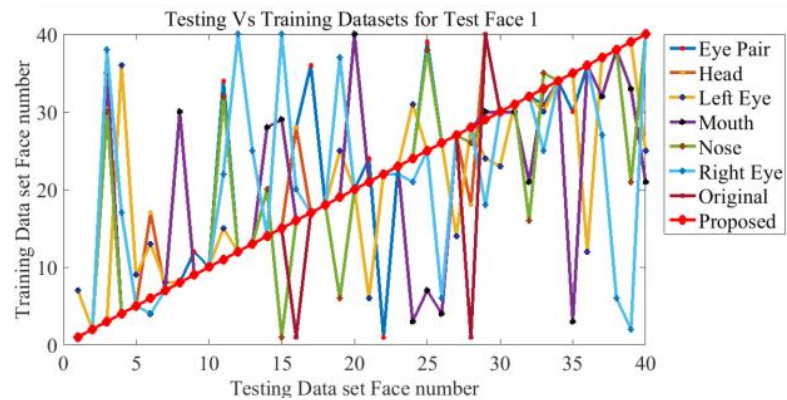

TABLE I

Fig. 26 Testing Vs Training Datasets for Face 1 test image.

Outputs of Different Face Recognition Algorithms for Test Face 1

\begin{tabular}{|c|c|c|c|c|c|c|c|c|}
\hline \multicolumn{9}{|c|}{ Face 1} \\
\hline 惫 & 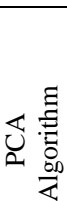 & 空芯 & 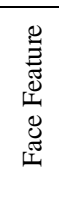 & 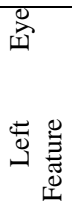 & 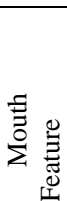 & $\begin{array}{l}\stackrel{0}{\Xi} \\
\stackrel{\Xi}{0} \\
0 \\
0 \\
0 \\
0 \\
Z\end{array}$ & 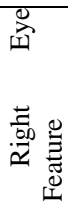 & 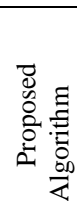 \\
\hline $\mathrm{S} 1$ & S1 & S1 & S1 & S7 & $\mathrm{S} 1$ & $\mathrm{~S} 1$ & $\mathrm{~S} 1$ & $\mathrm{~S} 1$ \\
\hline S2 & $\mathrm{S} 2$ & S2 & $\mathrm{S} 2$ & $\mathrm{~S} 2$ & S2 & S2 & S2 & $\mathrm{S} 2$ \\
\hline S3 & S3 & S3 & S3 & S3 & S35 & S30 & S38 & S3 \\
\hline S4 & S4 & S4 & S4 & S36 & S4 & S4 & $\mathrm{S} 17$ & S4 \\
\hline S5 & S17 & S5 & S5 & S9 & S17 & S5 & S5 & S5 \\
\hline S6 & S6 & S6 & S17 & S13 & S4 & S6 & S6 & S6 \\
\hline S7 & S7 & S7 & S7 & S8 & S7 & S7 & S7 & S7 \\
\hline S8 & S8 & S8 & S8 & S8 & S30 & S8 & S8 & S8 \\
\hline S9 & S22 & S12 & S29 & S22 & S9 & S9 & S29 & S29 \\
\hline S10 & S10 & S10 & S10 & S10 & S10 & S10 & S10 & S10 \\
\hline S11 & S11 & S34 & S11 & S15 & S11 & S32 & $\mathrm{S} 22$ & S11 \\
\hline S12 & S12 & S12 & $\mathrm{S} 12$ & S12 & $\mathrm{S} 12$ & S12 & $\mathrm{S} 40$ & $\mathrm{~S} 12$ \\
\hline S13 & S40 & S13 & S13 & S13 & $\mathrm{S} 40$ & S13 & S25 & $\mathrm{S} 13$ \\
\hline S14 & S14 & S14 & S14 & S14 & S28 & S20 & S14 & S14 \\
\hline S15 & S15 & S15 & S15 & S15 & $\mathrm{S} 29$ & S1 & $\mathrm{S} 40$ & S15 \\
\hline S16 & S1 & S28 & S28 & S1 & S16 & S16 & $\mathrm{S} 20$ & S16 \\
\hline S17 & S17 & S36 & S17 & S17 & S17 & S17 & S17 & S17 \\
\hline S18 & S18 & S18 & S18 & S18 & S18 & S18 & S18 & S18 \\
\hline S19 & S19 & S19 & S19 & S25 & S19 & S6 & S37 & S19 \\
\hline S20 & S22 & $\mathrm{S} 20$ & S20 & S22 & $\mathrm{S} 40$ & S20 & $\mathrm{S} 20$ & $\mathrm{~S} 20$ \\
\hline S21 & S21 & S24 & S21 & S6 & $\mathrm{S} 21$ & S21 & S21 & $\mathrm{S} 21$ \\
\hline S22 & S22 & S1 & S22 & S22 & S22 & S22 & S22 & $\mathrm{S} 22$ \\
\hline S23 & S23 & S23 & S23 & $\mathrm{S} 23$ & S23 & S23 & $\mathrm{S} 22$ & $\mathrm{~S} 23$ \\
\hline S24 & S24 & S24 & S24 & S31 & S3 & S24 & S21 & S24 \\
\hline S25 & S25 & S39 & S25 & S25 & S7 & S38 & S25 & $\mathrm{S} 25$ \\
\hline S26 & S26 & S26 & S26 & S26 & S4 & S26 & S6 & S26 \\
\hline S27 & S27 & S27 & S27 & S14 & S27 & S27 & S27 & S27 \\
\hline
\end{tabular}




\begin{tabular}{|l|l|l|l|l|l|l|l|l|} 
S28 & S28 & S28 & S18 & S28 & S28 & S26 & S28 & S28 \\
\hline S29 & S40 & S29 & S40 & S29 & S30 & S29 & S18 & S29 \\
\hline S30 & S30 & S30 & S30 & S23 & S30 & S30 & S30 & S30 \\
\hline S31 & S31 & S31 & S31 & S31 & S30 & S31 & S31 & S31 \\
\hline S32 & S32 & S32 & S32 & S32 & S21 & S16 & S32 & S32 \\
\hline S33 & S33 & S31 & S33 & S30 & S33 & S35 & S25 & S33 \\
\hline S34 & S1 & S34 & S1 & S40 & S40 & S34 & S40 & S40 \\
\hline S35 & S35 & S30 & S35 & S35 & S3 & S35 & S35 & S35 \\
\hline S36 & S36 & S36 & S36 & S12 & S36 & S36 & S36 & S36 \\
\hline S37 & S37 & S37 & S37 & S37 & S32 & S37 & S27 & S37 \\
\hline S38 & S38 & S38 & S38 & S38 & S38 & S38 & S6 & S38 \\
\hline S39 & S39 & S39 & S39 & S39 & S33 & S21 & S2 & S39 \\
\hline S40 & S40 & S40 & S40 & S25 & S21 & S40 & S40 & S40 \\
\hline
\end{tabular}

The proposed recognition engine is also verified on four different data sets available. The improvement in face recognition rate for all these data sets is listed in table.

The performance metrics for different algorithms shown below are with ORL database. All the performance curves show that irrespective of the algorithm, by changing the way of igniting the recognition engine, the performance of the algorithm is optimized. The proposed algorithm performed well and is shown in all the performance curves in blue lines.

\section{Performance curves of PCA Algorithm}

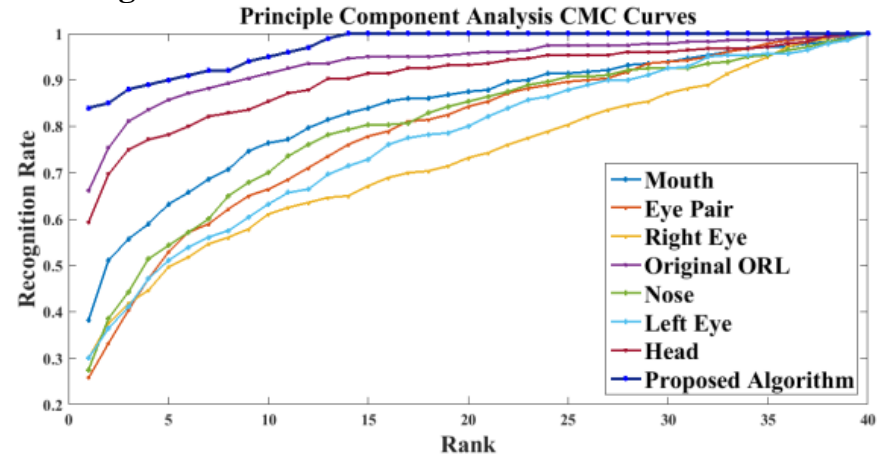

(a)

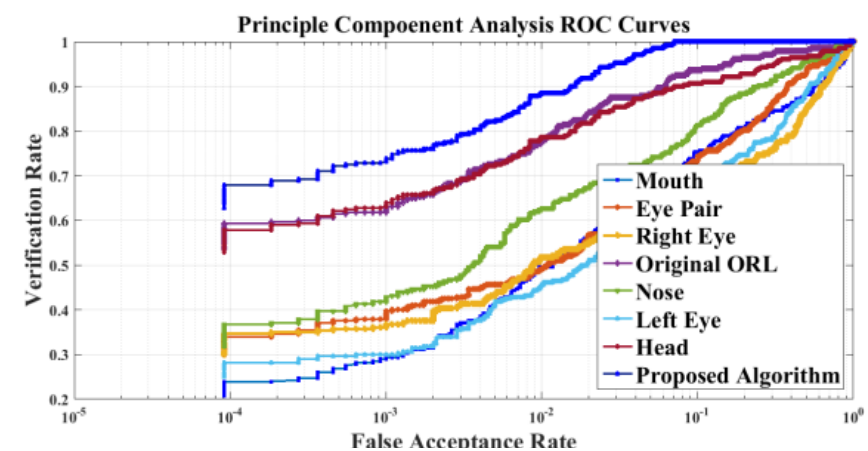

(b)

Fig. 27 (a) CMC and (b) ROC Curves of PCA Algorithm for Face, Mouth, Eye pair, right eye, left eye, nose and head data sets. The proposed algorithm is shown in blue.

\section{Conclusions}

In this paper, we have formulated a face partitioned algorithm and the decision making algorithm. A new and powerful way of igniting the recognition engine is introduced. This partition based ignition outperforms Eigen feature based face recognition algorithm. This ignition is verified on four other data sets. In all the data sets this method is performing well. Here, the face is divided into seven different face parts. Some of the face parts are redundant like right eye, left eye and eye pair for example. This redundancy is purposefully included in order to face the challenges like pose and illumination variations.

\section{TABLE II}

Different Datasets and their total number of images and persons

\begin{tabular}{|c|c|c|}
\hline Data base & Total number & Pose, \\
\hline
\end{tabular}
Total 


\begin{tabular}{|l|l|l|l|}
\hline & of persons & $\begin{array}{l}\text { Illumination and } \\
\text { facial expression } \\
\text { variations }\end{array}$ & $\begin{array}{l}\text { number } \\
\text { of face } \\
\text { images }\end{array}$ \\
\hline $\begin{array}{l}\text { Yale Database } \\
{[18]}\end{array}$ & 15 & 11 & 165 \\
\hline $\begin{array}{l}\text { Yale Face } \\
\text { Database 'B' } \\
{[19]}\end{array}$ & 10 & $\begin{array}{l}64 \text { illumination } \\
9 \text { poses }\end{array}$ & 5760 \\
\hline $\begin{array}{l}\text { BioID [20] - } \\
{[21]}\end{array}$ & 23 & $60-70$ & 1521 \\
\hline $\begin{array}{l}\text { Georgia Tech } \\
{[22]}\end{array}$ & 50 & 15 & 750 \\
\hline
\end{tabular}

\section{TABLE III}

Different Datasets and their total number of images and persons used in this experiment

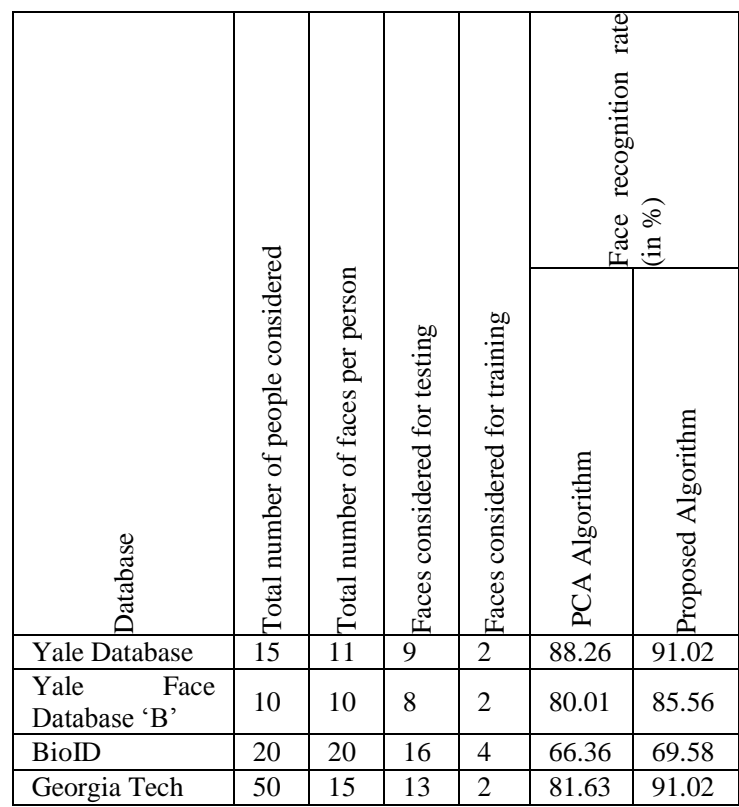

In case of testing images taken are more than one, then the face recognition rate is calculated by taking the average of the face recognition rates of all the testing images.

\section{References}

[1]. M. Turk and A. Pentland (Jun. 1991). "Face Recognition Using Eigenfaces." Proceedings of CVPR IEEE Computer Society. [Online]. pp. 586-591. Available: https://www.cs.ucsb.edu/ mturk/Papers/mturk-CVPR91.pdf.

[2]. Wenyi Zhao, Rama Chellappa, P Jonathon Phillips, "Subspace linear discriminant analysis for face recognition," Computer Vision Laboratory, Center for Automation Research, University of Maryland. Apr. 1994.

[3]. H. Wechsler, P. J. Phillips, V. Bruce, F. Fogelman Soulie and T. S. Huang (eds), "Face recognition: from theory to applications", NATO ASI series F, Computer and Systems Sciences, vol. 163, pp.446-456. 1998

[4]. Wenyi Zhao, Arvindh Krishnaswamy, Rama Chellappa, Daniel L Swets, and John Weng, "Discriminant analysis of principal components for face recognition," Face Recognition, Springer Berlin Heidelberg, pp. 73-85. 1998.

[5]. Sebastian Mika, Gunnar Ratsch, Jason Weston, Bernhard Scholkopf, and Klaus-Robert Muller. "Fisher Discriminant Analysis with Kernels.' [Online].

Available: http://citeseerx.ist.psu.edu/viewdoc/download;jsessionid=8B05D1BE9201C5AACD3A040ED70C4B48?doi=10.1.1.35.9904\&rep=rep $1 \&$ type $=$ pdf.

[6]. Hailing Zhou, Ajmal Mian, Lei Wei, Doug Creighton, Mo Hossny, and Saeid Nahavandi (December 2014). "Recent Advances on Single Modal and Multimodal Face Recognition: A Survey.”. [Online]. 44(6) pp.701-716. Available:

[7]. Gheorghita Ghinea, Raj kumar Kannan, and Suresh Kannaiyan (2014). "Gradient - Orientation - based PCA Subspace for Novel Face Recognition." IEEE Access. http://ieeexplore.ieee.org/xpl/abstractauthors.jsp?reload=true\&arnumber=6878464.

[8]. Navneet Dalal and Bill Triggs (Jun. 2005). "Histograms of Oriented Gradients for Human Detection." IEEE Computer Society Conference on Computer Vision and Pattern Recognition (CVPR'05). [Online]. 1, pp. 886-893 Available: https://lear.inrialpes.fr/people/triggs/pubs/Dalal-cvpr05.pdf.

[9]. Le An, Mehran Kafai, Bir Bhanu. "Face Recognition in Multi-Camera Surveillance Videos Using Dynamic Bayesian Network." [Online]. Available: www.ee.ucr.edu/ lan/papers/ICDSC12.pdf.

[10]. Paola Campadelli, Raffaella Lanzarotti, Chiara Savazzi. "A Feature-Based Face Recognition System." [Online]. Available: https://homes.di.unimi.it/ campadel/Articoli/ICIAP2003.pdf. 
[11]. Sasi Kumar Gurumurthy, B.K.Tripathy. (Jul. 2012). "Design and Implementation of Face Recognition System in MATLAB using the Features of Lips.” International Journal of Intelligent Systems and Applications. [Online]. (8) pp.30-36. Available: www.mecspress.org/ijisa/ijisa-v4-n8/IJISA-V4-N8-4.pdf.

[12]. Ognjen Arandjelovic, "Learnt quasi-transitive similarity for retrieval from large collections of faces", arXiv preprint arXiv. 2016.

[13]. Paul Viola and Michael J. Jones (Jul. 2001). "Robust Real-Time Object Detection.” Second International Workshop on Statistical and Computational Theories of Vision - Modeling, Learning, Computing, and Sampling. [Online]. 4. Available: https://www.cs.cmu.edu/ efros/courses/lbmv07/papers/viola-ijcv-01.pdf

[14]. Samy Bengio and Johnny Mari'ethoz. "The Expected Performance Curve: a New Assessment Measure for Person Authentication." [Online]. Available: publications.idiap.ch/downloads/reports/2004/bengio_2004_odyssey.pdf.

[15]. Samy Bengio, Johnny Mari'ethoz, and Mikaela Keller. "The Expected Performance Curve." [Online]. Available: bengio.abracadoudou.com/publications/pdf/bengio_2005_icml.pdf.

[16]. Seung-Jean Kim, Alessandro Magnani, and Stephen Boyd. "Optimal Kernel Selection in Kernel Fisher Discriminant Analysis." [Online].Available: www.autonlab.org/icml_documents/.../059_Optimal_Kernel_Selec.pdf.

[17]. ORL Database. AT\&T Laboratories, Cambridge http://www.cl.cam.ac.uk/Research/DTG/attarchive:pub/data/att_faces.zip.

[18]. Yale face database. [Online]. Available:http://vision.ucsd.edu/datasets/yale_face_dataset_original/yalefaces.zip.

[19]. Yale face B database. [Online]. Available: http://vision.ucsd.edu/ iskwak/extyaledatabase/extyaleb.html.

[20]. O. Jesorsky, K. Kirchberg, R. Frischholz, In J. Bigun and F. Smeraldi, editors. "Face Detection Using the Hausdorff Distance." Audio and Video based Person Authentication - AVBPA 2001, pages 90-95. Springer, 2001. [Online]. Available: https://www.bioid.com/download?path=AVBPA01BioID.pdf.

[21]. Bio ID Face Database. [Online]. Available: https://www.bioid.com/About/BioID-Face-Database

[22]. Georgia Tech Face Database. [Online]. Available: http://www.anefian.com/research/gt_db.zip.

[23]. Harihara Santosh Dadi and P G Krishna Mohan, "Performance Evaluation of Eigen faces and Fisher faces with different pre-processed Data sets," International Journal of Advanced Research in Computer Engineering \& Technology (IJARCET), vol. 4, no. 5, pp. 2110 2116. May 2015.

[24]. Harihara Santosh Dadi and P G Krishna Mohan, "Enhancement of Face Recognition Rate by Data Base Pre-processing," International Journal of Computer Science and Information Technologies, IJCSIT, vol. 6, no. 3, pp. 2978-2984. Jun. 2015.

Mr. D. Harihara Santosh obtained his B. Tech. and M. Tech degrees from JNT University, Hyderabad in the

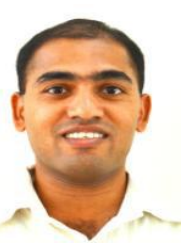
year 2005 and 2010. Presently he is pursuing Ph.D, in Video Processing at JNTU, Hyderabad. $\mathrm{He}$ is presently pursuing his Ph.D. under the Guidance of Dr. P.G. Krishna He has 13 publications in both International and National Journals and presented 22 papers at various International and National Conferences. His areas of interests are Image and Video Processing.

Dr.P.G.Krishna Mohan presently working as Professor in Institute of Aeronautical College of Engineering,

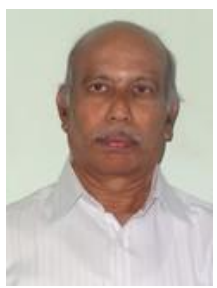
Hyderabad. He Worked as Head of ECE Dept. , Member of BOS for ECE faculty at University Level, Chairman of BOS of EIE group at University level, Chairman of BOS of ECE faculty for JNTUCEH, Member of selection committees for Kakitiya, Nagarjuna University, DRDL and convener for Universite a Hidian committees. He has more than 48 papers in various International and National Journals and Conferences. His areas of interests are Signal Processing, Communications. 